\title{
El espacio público como eje de integración social y escenario de la vida ciudadana
}

\author{
María del Rosario Santos-De Aguirre*, Juan Pimiento-Osorio ${ }^{\star \star}$
}

\footnotetext{
Abogada. Profesora asociada investigadora, miembro del grupo de investigación CIP-URIS y directora del semillero de investigación Ecos, Escuela de Derecho, Facultad de Derecho, Universidad Pontificia Bolivariana, Bucaramanga, Colombia. Correo electrónico: maria.santos@upb.edu.co

** Abogado. Asesor del Centro de Conciliación, investigador y monitor de investigaciones, Escuela de Derecho, Facultad de Derecho, Universidad Pontificia Bolivariana, Bucaramanga, Colombia.

Correo electrónico: juan.pimiento@upb.edu.co
}

Recibido: 10 de junio del 2015 Aprobado: 27 de julio del 2015

Cómo citar este artículo: María del Rosario Santos-De Aguirre; Juan Pimiento-Osorio. El espacio público como eje de integración social y escenario de la vida ciudadana. DIXI 22. Diciembre 2015. Pág. 87. doi: http://dx.doi.org/10.16925/ di.v17i22.1240

\section{Resumen}

Introducción: gracias a la Constitución Política de Colombia de 1991, se ha repensado en el país el espacio público desde la óptica jurídica. Asimismo, desde las administraciones territoriales, como es el caso de Bucaramanga, se han trazado políticas públicas para su reconocimiento, protección y recuperación. No obstante, el actuar del ejecutivo local no ha sido encauzado a criterios del todo constitucionales, ni tampoco estos responden a una valoración de la prerrogativa colectiva superior a su aspecto material. Metodología: tal propuesta surge a través del estudio acucioso de las disposiciones en Derecho que regulan el espacio público, contrastado con su valoración en tendencias de formación ciudadana desde lo público y en urbanismo, para así partir de un concepto amplio. Luego, por intermedio de encuestas, se procuró reconocer el ideario acerca del espacio público de actores fundamentales en su utilización en el municipio de Bucaramanga, evento que significó la interlocución con la autoridad administrativa a fin de reconocer la política pública en la materia y alcanzar una posición desde la academia que incluya una crítica sobre el trabajo realizado y permita valorar desde una nueva perspectiva el espacio público. Resultados y conclusiones: por lo anterior, la academia, representada por la Facultad de Derecho de la Universidad Pontificia Bolivariana, propone una visión más amplia del espacio público, en la cual concurran aspectos suprajurídicos que resulten afines a la percepción social que se tiene por dicho derecho y que, además, resulte respetuosa y de un constitucionalismo con rasgos humanos.

Palabras clave: espacio público, constitucionalismo, derechos colectivos, participación ciudadana, política pública, urbanismo. 


\title{
The Public Space as Backbone for Social Integration and as the Scenario of Civic Life
}

\begin{abstract}
Introduction: Thanks to the 1991 Political Constitution of Colombia, the public space has been re-examined from the legal perspective. At the same time, territorial administrations, such as Bucaramanga, have created public policies for its recognition, protection and recovery. Nonetheless, actions by local administrators have not been guided by fully constitutional criteria, nor are they based on evaluation of the collective prerogative that is superior to its material aspect. Methodology: The proposal stems from a careful study of legal provisions that regulate the public space, contrasted with its valuation in civic education trends from the public perspective and that of urbanism, as a broad basis. Then, using surveys, the set of ideas about the public space applied by basic actors in its use in the municipality of Bucaramanga is recognized. This signified interlocution with the administrative authority to recognize public policy in the matter and reach a consensus in academia that would include a critique of the work carried out while facilitating valuation of the public space from a new perspective. Results and Conclusions: Given the above, academia, represented by the Law Faculty of the Universidad Pontificia Bolivariana, proposes a broader vision of the public space, in which there are supralegal aspects that are in harmony with social perception about that right and which also are respectful of, and have a constitutionalism with, human features.
\end{abstract}

Keywords: public space, constitutionalism, collective rights, civic participation, public policy, urbanism.

\section{O espaço público como eixo de integração social e cenário da vida cidadã}

\section{Resumo}

Introdução: graças à Constituição Política da Colômbia de 1991, repensou-se no país o espaço público a partir da ótica jurídica. Igualmente, a partir das administrações territoriais, como é o caso de Bucaramanga, traçaram-se políticas públicas para seu reconhecimento, proteção e recuperação. No entanto, a ação do executivo local não foi direcionada por critérios totalmente constitucionais, nem estes respondem a uma valorização da prerrogativa coletiva superior a seu aspecto material. Metodologia: essa proposta surge por meio do estudo minucioso das disposições em Direito que regulam o espaço público, contrastado com a valorização deste em tendências de formação cidadã a partir do público e em urbanismo, para assim partir de um conceito amplo. Em seguida, por meio de entrevistas, procurou-se reconhecer o ideário acerca do espaço público de atores fundamentais em sua utilização no município de Bucaramanga, evento que significou a interlocução com a autoridade administrativa a fim de reconhecer a política pública na matéria e alcançar uma posição da academia que inclua uma crítica sobre o trabalho realizado e permita valorizar o espaço público a partir de uma nova perspectiva. Resultados e conclusões: portanto, a academia, representada pela Faculdade de Direito da Universidade Pontifícia Bolivariana, propõe uma visão mais ampla do espaço público, na qual coincidam aspectos suprajurídicos que estejam relacionados com a percepção social que se tem desse direito e que, além disso, seja respeitosa e decorra de um constitucionalismo com características humanas.

Palavras-chave: espaço público, constitucionalismo, direitos coletivos, participação cidadã, política pública, urbanismo. 


\section{INTRODUCCIÓN}

El presente documento recoge la labor investigativa realizada por docentes y estudiantes de la Facultad de Derecho de la Universidad Pontificia Bolivariana, seccional Bucaramanga, durante el tiempo comprendido entre el 2011 y el 2013, frente a la temática del espacio público en el municipio de Bucaramanga.

La iniciativa surge de una investigación anterior, realizada por las docentes de la facultad Úrsula Fernanda Castellanos y María Del Rosario Santos, quienes profundizaron sobre acciones públicas de orden constitucional utilizadas para la salvaguardia de derechos colectivos, correspondiente a la línea del Neoconstitucionalismo. Así, se realizó el estudio local sobre el impacto de tales instrumentos, en ese momento reconocidos desde la norma positiva como acciones populares y de grupo, en la protección de los derechos constitucionales de tercera generación. Allí se observa preliminarmente el valor que tiene para los habitantes del municipio una real protección del derecho al espacio público.

Partiendo del estudio en referencia, se identifica como problema de investigación la congruencia entre los conceptos jurídicos, las propuestas administrativas, el interés colectivo y el valor real del espacio público en el municipio, y la posterior expedición del Decreto 0179 de 2012. En este sentido existiría una necesaria fricción entre los diferentes sectores sociales, en cuanto el mentado acto administrativo que fijaría una política pública con respecto al derecho colectivo señalado, como también proveería de facultades especiales a la autoridad para hacer respetar tal prerrogativa. Con el propósito de profundizar la propuesta investigativa de la facultad y de posicionarla como un verdadero referente conceptual al momento de abordar la problemática del espacio público, se decide confrontar primariamente el ideal constitucional, alimentado por múltiples interpretaciones de la Corte Constitucional, con la identidad que se da al derecho colectivo en la sociedad bumanguesa y en sus burgomaestres. Como consecuencia de lo anterior, se fija como pregunta de investigación la siguiente: ¿cómo construir el espacio público como eje integrador social y escenario de la vida ciudadana?

A fin de garantizar una respuesta acorde con el Derecho del anterior interrogante, así como en procura de diseñar una propuesta conceptualmente correcta que refleje la verdadera interacción social que deviene de la utilización del espacio público, se toman como primer elemento conceptualizador del Derecho las descripciones jurídicas que se dan de este, identificando su reconocimiento legal, constitucional y jurisprudencial, para así garantizar una visión holística de lo que realmente es el espacio público. A su vez, se nutre la noción esgrimida desde la investigación con las valoraciones que desde el urbanismo y la literatura de participación ciudadana se dan para el fenómeno tratado.

Ese primer matiz conceptual es posteriormente comparado con la valoración que se hace también del espacio público en la sociedad misma, con quienes interactúan cotidianamente en dicho espacio y con la postura de la autoridad administrativa, quien regularía con aparente mayor profundidad la temática. Para ello se diseñaron diversos instrumentos de recolección de información que serán debidamente presentados en el acápite de metodología de la investigación del presente documento. A partir de la labor investigativa enfocada a través de los dos frentes enunciados, podrá el lector comprender a plenitud las conclusiones arribadas desde la investigación acometida.

\section{INSTRUMENTOS}

\section{A. Marco teórico}

\section{El espacio público desde la óptica del derecho}

La pregunta de investigación propuesta responde a una pretensión de enriquecer el valor del espacio público en la sociedad, no obstante la estructuración de una propuesta real para ello debe partir y desarrollarse desde los límites jurídicos, sociales, culturales y políticos que regentan directrices sobre la materia. La búsqueda de espacios de integración social desde lo público conlleva consecuencialmente el reconocimiento primario de lo que la ley considera realmente como espacio público.

La Ley 9 de 1989, por la cual se dictan normas sobre planes de desarrollo municipal, compraventa y expropiación de bienes, en su artículo 5 esboza un primer concepto de espacio público. El legislador en dicha ocasión optó por señalar qué elementos o estructuras físicas deben valorarse como espacio público, partiendo así de una concepción exclusivamente material de la mentada prerrogativa. El criterio básico utilizado es el de destinación de un bien. Así, si esta se da para la satisfacción de necesidades urbanas 
colectivas que trascienden los intereses individuales de los habitantes, se entenderá como espacio publicó la estructura aludida. Dicha disposición enuncia adicionalmente como espacio público los siguientes:

- Las áreas requeridas para la circulación tanto peatonal como vehicular (vías públicas) - como por ejemplo calles, plazas, puentes y caminos -

- Las áreas para la recreación pública, activa o pasiva - léase estadios, parques y zonas verdes, por ejemplo-.

- Las franjas de retiro de las edificaciones sobre las vías -es decir andenes o demás espacios peatonales-.

- Las fuentes de agua y las vías fluviales que no son objeto de dominio privado.

- Las áreas necesarias para la instalación y el mantenimiento de los servicios públicos básicos o para la instalación y el uso de los elementos constitutivos del amueblamiento urbano en todas sus expresiones.

- Las áreas para la preservación de las obras de interés público y de los elementos históricos, culturales, religiosos, recreativos y artísticos, para la conservación y preservación del paisaje.

- Los elementos naturales del entorno de la ciudad.

- Lo necesario para la preservación y conservación de las playas marinas y fluviales, los terrenos de bajamar, así como la de sus elementos vegetativos, arenas y corales.

- En general, todas las zonas existentes o debidamente proyectadas en las que el interés colectivo sea manifiesto y conveniente y que constituyen por consiguiente zonas para el uso o el disfrute colectivo. ${ }^{1}$

Con la entrada en vigencia de la Constitución Política de Colombia de 1991 y su posterior readecuación interpretativa de todas las disposiciones legales vigentes que no le sean contrarias, se observa una expansión desde la órbita jurídica de lo que se esbozó antes como espacio público. En el capítulo 3 del título II de la norma superior, se conciben como derechos colectivos el ambiente sano y el espacio público. Para el primero, garantiza a la población su disfrute y la participación en las decisiones que le afecten. Para el segundo, el constituyente realza la obligación de preservar en su integralidad dicho derecho, señalando además como principio básico el de la prevalencia del interés general al momento de decidir sobre estructuras que en apariencia se conciban desde la descripción legal como espacio público. También la Constitución

1. Véase Ley 9 de 1989. Por la cual se dictan normas sobre planes de desarrollo municipal, compraventa y expropiación de bienes y se dictan otras disposiciones. Noviembre 1 de 1989 . Do N. ${ }^{\circ} 38.650$. suministra a los ciudadanos acciones públicas eminentemente constitucionales de salvaguardia de sus derechos colectivos, actualmente denominados medios de control que encuentran debida regulación en la Ley 1437 de 2011.

El segundo derecho colectivo señalado -que es el que atañe a la presente investigación- encuentra desarrollo jurisprudencial de mano de la Corte Constitucional, como guardiana de la Constitución, en diversas oportunidades. Al respecto, la corporación en sentencia de tutela número 908 de 2010 manifestó lo siguiente:

La Constitución de 1991, al darle relevancia constitucional al buen y adecuado uso del espacio público, procura garantizar que los colombianos gocen en condiciones de igualdad, de todos los espacios que son de uso común o público, con el fin de permitir el goce de derechos tales como el de circulación, seguridad, accesibilidad y medio ambiente. ${ }^{2}$

De tal declaración, se entrevé que el espacio público, aunque se garantice en su existencia material, supone que los usuarios de la administración le brinden un uso adecuado a fin de obtener de él todos los derechos que les son propios.

Así, la dimensión del Derecho se eleva a una verdadera plataforma de reconocimiento social y de interacción, como si se tratara de un vehículo que transporta los intereses de los individuos para que, aunque sea por un momento, confluyan con los que tienen los otros. Tal como fue reflexionado por el guardián constitucional en el 2000, bajo providencia de tutela 024 :

En el mejoramiento de la calidad de vida y en la misma protección a la vida, el manejo del espacio público en las zonas urbanas (donde hoy vive la mayor parte de la humanidad) es más que un tema urbanístico, ha llegado a ser tema del constitucionalismo con rasgos humanos. Ese manejo está influenciado por el tráfico en la ciudad, problema éste que constituye uno de los más delicados en la sociedad moderna porque el transporte urbano alteró el cuadro tradicional de las áreas locales y el desplazamiento de los peatones. ${ }^{3}$

Se observa que desde principios del milenio el espacio público cobró un papel preponderante en la estructuración de una sociedad sana. Por lo tanto, no

2. Véase Corte Constitucional de Colombia. Sentencia T-908/10. (M. P. Mauricio González Cuervo; Noviembre 20 de 2010).

3. Véase Corte Constitucional de Colombia. Sentencia T-024/oo. (M. P. Alejandro Martínez Caballero). (Las negrillas son añadidas). 
sólo las autoridades legislativas, sino las judiciales y también las administrativas deben emprender todas las acciones que estuviesen a su haber con el fin de preservar tal circunstancia y así garantizar todas las prerrogativas que de ella se desprendan.

La perspectiva en que se aborda la acción de "construir" es abstracta, pues el objeto de tal verbo para la presente propuesta es inmaterial. Se circunscribe a la participación de los individuos en el entorno comunitario, no a la edificación de alguna estructura en particular. Esto como respuesta a la búsqueda de un espacio público integrador de la población. De esta forma, surge la preocupación de identificar las formas adecuadas en que el colectivo consiga participar activamente en la vida común que surge de la necesaria interacción que se presenta en zonas concretas.

Si las personas tienen las condiciones para participar activa y comprometidamente en la construcción de su entorno, sin duda no solo se fortalecen los nexos de apropiación y pertenencia por una ciudad de todos, "hecha por todos", sino que se promueven al acercamiento y la comunicación entre los distintos actores tanto individuales como colectivos comprometidos con la transformación y consecuente apropiación de la misma. ${ }^{4}$

\section{El espacio público como escenario político}

Idea concebida a partir de una noción abstracta de espacio público, entendiéndolo como la ciudad misma, el cual comprende no sólo las zonas comunitarias (físicamente comprobables), sino también todo el espectro de posibilidades de interacción ciudadana en una municipalidad específica.

Interacción que no sólo es posible, es una verdadera prerrogativa de toda persona frente al municipio en que reside. Se erige así el derecho a la ciudad o condición ciudadana material como la posibilidad de participar activamente de la oferta pública de lo público en cualquier municipio, concepto afín al expuesto por Yory y Delgado en la introducción de su investigación, en la cual se insta a concebir al espacio público como "un escenario pedagógico de construcción de ciudadanía; es decir, de apropiación

4. Véase Carlos Mario Yory y Ricardo Delgado. Espacio público Y FORMACIÓN DE CIUDADANÍA: DESAFÍOS Y PROPUESTAS PARA LA CONSTRUCCIÓN DE UN PROYECTO CONCERTADO DE CIUDADANÍA APTO PARA LA CIUDAD LATINOAMERICANA A PARTIR DE LA APROPIACIÓN RESPONSABLE DEL ESPACIO PÚBLICO. Pontificia Universidad Javeriana. (2007). de la ciudad a partir de la directa intervención en la construcción-transformación de su espacio público."5

Por lo tanto, es público adicionalmente a lo que reciba esa denominación jurídica - condición especialísima-, todo aquello que la comunidad utilice bajo intereses colectivos, mucho más cuando estos sean direccionados a la construcción de una amigable sociedad. Así, "el espacio público como legítimo escenario de socialización, de construcción de comunidad, por tanto no solo llamado a ser preservado sino a hallarse en garantía de todas las herramientas propias de su construcción óptima e incluyente frente a todos los sujetos que intervengan en él.

Más que una gran valla publicitaria, el espacio público debe construirse como el principal y más significativo canal de disertación política de cualquier municipalidad.

De esta manera, es necesario resaltar lo que menciona José Miguel Pereira: "Lo público no se reduce a lo estatal. Hoy constituye multiplicidad de espacios de interacción, en los cuales la acción del ciudadano, a través de la comunicación, busca identificar intereses colectivos públicos." Por tal motivo, no se debe diferenciar o contraponer lo público de lo privado, pues estas se consolidan como la base de una estructura única e inseparable.

\section{B. Metodología}

Desde el diseño de la metodología se identificó el municipio de Bucaramanga, excluyendo su área metropolitana, como espacio dentro del cual se iniciaría la actividad investigativa. Esta se circunscribía a un carácter eminentemente teórico, pues a través de ella se valorarían prioritariamente fuentes formales del Derecho, extendiéndose la recolección de información a la interlocución con actores importantes frente al ejercicio de identificación del espacio público. Asimismo, la investigación se caracterizó bajo un corte cualitativo y un enfoque interpretativo, en los cuales - desde la hermenéutica - se analizaron y calificaron los diferentes fenómenos confluyentes sobre la problemática enunciada.

Las fuentes del Derecho a las que acudió la investigación fueron específicamente la jurisprudencia y la normatividad local, a fin de contrastar la eficacia de los mandatos de las autoridades, la preservación de los derechos constitucionales de los ciudadanos, la efectiva protección del espacio público 
y la postura de la comunidad con respecto a la actividad del Estado.

Se denominó el crisol en el que se fraguaron los resultados como "óptica jurídico-social", pues con la investigación se pretendió presentar la forma en que las decisiones administrativas y judiciales modifican los roles que desempeñen los individuos en la sociedad, en relación con la regulación que el Derecho realiza de la utilización del espacio público en la ciudad de Bucaramanga. Tales resultas engloban análisis jurisprudenciales, seguimiento del desempeño en la ejecución de las diversas políticas, planes, programas y proyectos públicos, entrevistas a servidores públicos con funciones relevantes al espacio público y encuestas. Estas últimas permiten visibilizar cifras porcentuales de las diferentes percepciones que puede concebir la comunidad bumanguesa con respecto a la política de preservación del espacio público de Bucaramanga y sus repercusiones sociales.

\section{Resultados}

Los instrumentos empleados para llevar a cabo el desarrollo de la investigación son los siguientes.

\section{A. Encuestas}

Con el propósito de reconocer la percepción existente en la población bumanguesa respecto al espacio público y frente al fenómeno del comercio informal y las ventas ambulantes, se diseñaron instrumentos de recolección de información figurativos en encuestas, a través de los cuales se consultaron 40 vendedores formales, 167 transeúntes y 89 vendedores informales de las zonas del municipio en que es más visible la confluencia de dichos actores. Se realizaron jornadas a lo largo de la carrera 33 y el Paseo del Comercio.

\section{B. Interacción con la autoridad}

A partir del diálogo establecido con la autoridad administrativa local, se obtuvo documento contentivo de las ejecutorias de la política de espacio público del municipio y de la actuación del comité interinstitucional de recuperación, protección y defensa del espacio público. Igualmente se entrevistó al abogado Kadir Pilonieta, defensor del espacio público del municipio de Bucaramanga. Entrevista realizada en el 2012.

\section{Percepción de la comunidad}

Ante la pregunta, ¿qué se entiende por espacio público?, no existe un consenso por parte de los tres sectores entrevistados: transeúntes, vendedores informales y vendedores formales. Por su parte, los primeros consideran tal prerrogativa prioritariamente como un lugar de movilización, un corredor vial; un buen porcentaje de tales personas manifestó entender el espacio público como un lugar de recreación.

\section{¿QUÉ ENTIENDE POR ESPACIO PÚBLICO?}

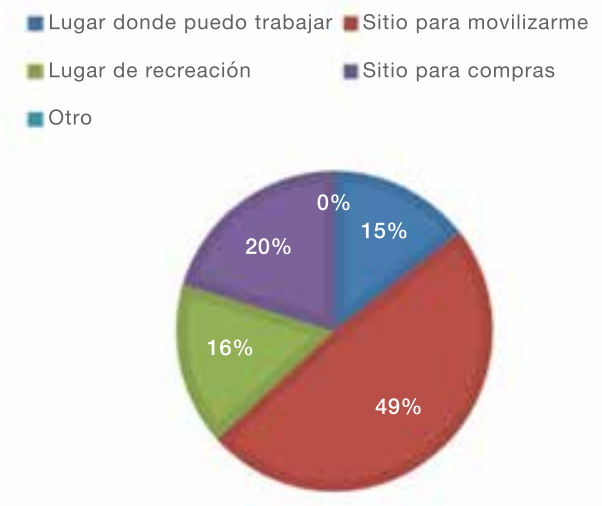

Gráfico 1. ¿Qué entiende por espacio público? Fuente: elaboración propia a partir de encuesta de la investigación.

De manera dispar, los comerciantes formales consideran en mayor medida que la opción de corredor vial es la que mejor define el derecho. También son el grupo que en mayor medida observa que la limitación al espacio público se da prioritariamente por la puesta en marcha de ventas ambulantes.

\section{¿QUÉ ENTIENDE POR ESPACIO PÚBLICO?}
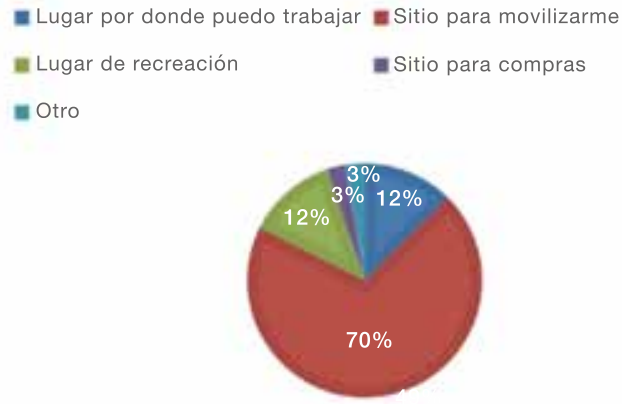

Gráfico 2. ¿Qué entiende por espacio público? Fuente: elaboración propia a partir de encuesta de la investigación. 


\section{¿QUÉ ENTIENDE POR OCUPACIÓN DEL ESPACIO PÚBLICO?}
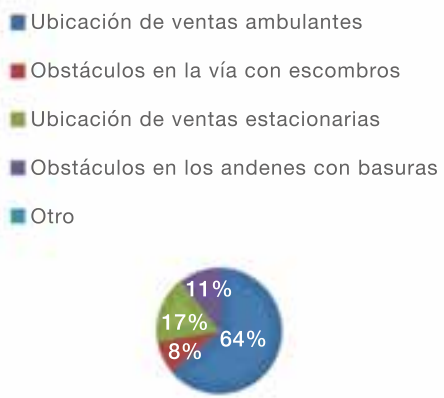

Gráfico 3. ¿Qué entiende por ocupación del espacio público? Fuente: elaboración propia a partir de encuesta de la investigación.

Por su parte, los comerciantes informales en su mayoría entienden el espacio público como un lugar en que se puede trabajar libremente.

\section{¿Qué entiende por Espacio público?

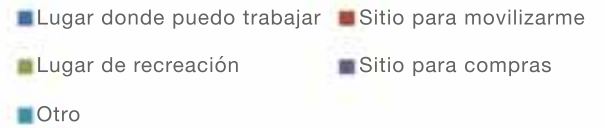

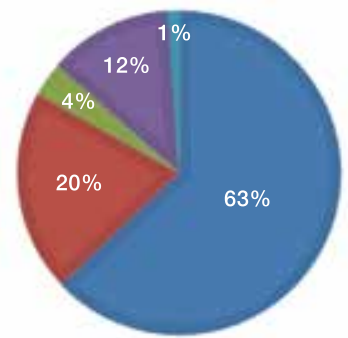

Gráfico 4. ¿Qué entiende por ocupación del espacio público? Fuente: elaboración propia a partir de encuesta de la investigación.

No obstante, un número mayoritario de encuestados, sin distinción del grupo al que pertenecieran, guarda una interpretación tradicionalista de lo que es el espacio público, al observarlo exclusivamente como un sitio de movilización.

En los instrumentos figurativos de encuestas no existieron otras preguntas que ahondaran sobre el imaginario colectivo alrededor del derecho colectivo desarrollado.

\section{Postura del municipio}

El Decreto 0179 de 2012, expedido por el alcalde de Bucaramanga, dicta disposiciones en relación con la recuperación y preservación del espacio público $\mathrm{y}$, aunque en su seno crea el comité de recuperación, protección y preserva de la misma prerrogativa, no dispone de un concepto claro para la ciudadanía sobre lo que es el espacio público. Esto significa que necesariamente la legislación nacional vigente será la que lo determine, es decir, que dicha política y todas las demás que le sean complementarias se utilizan exclusivamente para lo que se denominó a lo largo del presente artículo como "concepción tradicionalista del espacio público".

El acto administrativo señalado responde, en su mayoría, a la institución de un debido proceso para aquellos casos en los cuales se pretende la recuperación de un lugar de aparentes características físicas de orden público. Tal hecho se observó durante la entrevista realizada a Kadir Pilonieta, quien en ese entonces fungía como defensor del espacio público. Allí manifiesta cómo la política pública del municipio está encaminada a formalizar el comercio realizado por vendedores ambulantes durante los últimos años, pero no está referida a la readecuación de dichos espacios a eventos sociales verdaderamente públicos, es decir, como un lugar en que la sociedad se ve necesariamente llamada a ser una. Así, la concepción constitucional expresada en el marco teórico de la presente disertación no se desarrolla plenamente, pues la autoridad no conduce al ciudadano, ni tampoco brinda un escenario propicio para el desarrollo comunitario en conjunto.

\section{Conclusiones}

Las decisiones administrativas tomadas desde el municipio de Bucaramanga en desarrollo del Decreto 0179 de 2012, aunque legales, no desarrollan a plenitud el derecho colectivo al espacio público. Esto en cuanto que minimizan la esfera de acción al campo de la recuperación y desconocen dicha prerrogativa como un criterio de calidad de vida de la población.

Por otra parte, la muestra entrevistada de población bumanguesa restringe de cierta manera su concepción del espacio público a aspectos netamente urbanísticos, pues reduce su noción a la destinación tradicional que se da a este. No lo perciben como un escenario en el que la sociedad está llamada a desarrollarse, sino como el lugar en que inevitablemente los individuos se encuentran.

La administración municipal, mediante la Secretaría de Gobierno y demás agencias que la complementan a nivel local, ha considerado la invasión del espacio público por parte del comercio informal 
y formal como un verdadero problema social, en cuanto ello impide la adecuada utilización de tal derecho colectivo, el cual resulta ser principalmente el tránsito vehicular y peatonal.

Por último, como medida paliativa para lo que serían las consecuencias directas a la porción de la comunidad que utiliza el espacio público como plataforma de trabajo, la autoridad con anterioridad a la ejecución del acto administrativo desarrolló sistemas de publicidad y foros de participación ciudadana, a fin de construir una alternativa comercial para dichas personas, hecho que resultó significativamente complejo y que significó la continua modificación de la fecha de puesta en marcha de las actividades policiales de preservación del espacio público.

\section{REFERENCIAS}

Constitución Política de Colombia [Const]. (Colombia).

Corte Constitucional de Colombia. Sentencia T-908/10. (M. P. Mauricio González Cuervo; Noviembre 12 de 2010).

Ley 9 de 1989. Por la cual se dictan normas sobre planes de desarrollo municipal, compraventa y expropiación de bienes y se dictan otras disposiciones. Noviembre 1 de 1989 . Do N. ${ }^{\circ} 38.650$.
Mario Yory y Ricardo Delgado. EsPaCio PÚbLICO Y FORMACIÓN DE CIUDADANÍA: DESAFÍOS Y PROPUESTAS PARA LA CONSTRUCCIÓN DE UN PROYECTO CONCERTADO DE CIUDADANÍA APTO PARA LA CIUDAD LATINOAMERICANA A PARTIR DE LA APROPIACIÓN RESPONSABLE DEL ESPACIO PÚBlico. Pontificia Universidad Javeriana. (2007)

Úrsula Fernanda Castellanos Moreno. EFICACIA DE LAS ACCiones de grupo en el Área Metropolitana de BUCARAMANGA CON RESPECTO AL MEDIO AMBIENTE. Universidad Pontificia Bolivariana. (2011).

Julio Enrique González Villa. Derecho ambiental coLombiano. Tomo I y II. Universidad Externado de Colombia. (2006).

Beatriz Toro Londoño, Gloria Amparo Rodríguez y Herbert Herrera Carrascal. Perspectivas del Derecho ambiental en Colombia. Universidad del Rosario. (2006)

Pedro Páramo y Mónica Eliana García Gil. La Dimensión Social del espacio público. Aportes Para la CaLIDAD DE VIDA URbana. Universidad Pedagógica Nacional. (2010).

María del Rosario Santos. EFICACIA DE LAS ACCIONES POPULARES EN El Área METropolitana de BuCARAMaNGA CON RESPECTO AL MEDIO AMBIENTE. Universidad Pontificia Bolivariana. (2011). 